\title{
Radio emission of highly inclined cosmic ray air showers measured with LOPES
}

J. Petrovic ${ }^{1}$, W. D. Apel ${ }^{2}$, T. Asch ${ }^{3}$, F. Badea ${ }^{2}$, L. Bähren ${ }^{4}$, K. Bekk ${ }^{2}$, A. Bercuci ${ }^{5}$, M. Bertaina ${ }^{6}$, P. L. Biermann ${ }^{7}$, J. Blümer ${ }^{2,8}$, H. Bozdog ${ }^{2}$, I. M. Brancus ${ }^{5}$, M. Brüggemann ${ }^{9}$, P. Buchholz ${ }^{9}$, S. Buitink ${ }^{1}$, H. Butcher ${ }^{4}$, A. Chiavassa ${ }^{6}$, F. Cossavella ${ }^{8}$, K. Daumiller ${ }^{2}$, F. Di Pierro ${ }^{6}$, P. Doll ${ }^{2}$, R. Engel $^{2}$, H. Falcke ${ }^{1,4,7}$, H. Gemmeke ${ }^{3}$, P. L. Ghia ${ }^{10}$, R. Glasstetter ${ }^{11}$, C. Grupen ${ }^{9}$, A. Haungs ${ }^{2}$, D. Heck ${ }^{2}$, J. R. Hörandel ${ }^{8}$, A. Horneffer ${ }^{1}$, T. Huege ${ }^{2}$, K.-H. Kampert ${ }^{11}$, Y. Kolotaev ${ }^{9}$, O. Krömer ${ }^{3}$, J. Kuijpers ${ }^{1}$, S. Lafebre ${ }^{1}$, H. J. Mathes ${ }^{2}$, H. J. Mayer ${ }^{2}$, C. Meurer ${ }^{2}$, J. Milke ${ }^{2}$, B. Mitrica ${ }^{5}$, C. Morello ${ }^{10}$, G. Navarra ${ }^{6}$, S. Nehls ${ }^{2}$, A. Nigl ${ }^{1}$, R. Obenland ${ }^{2}$, J. Oehlschläger ${ }^{2}$, S. Ostapchenko ${ }^{2}$, S. Over ${ }^{9}$, M. Petcu ${ }^{5}$, T. Pierog ${ }^{2}$, S. Plewnia ${ }^{2}$, H. Rebel ${ }^{2}$, A. Risse ${ }^{13}$, M. Roth ${ }^{2}$, H. Schieler ${ }^{2}$, O. Sima ${ }^{5}$, K. Singh ${ }^{1}$, M. Stümpert ${ }^{8}$, G. Toma ${ }^{5}$, G. C. Trinchero ${ }^{10}$, H. Ulrich ${ }^{2}$, J. van Buren ${ }^{2}$, W. Walkowiak ${ }^{9}$, A. Weindl ${ }^{2}$, J. Wochele $^{2}$, J. Zabierowski ${ }^{13}$, J. A. Zensus ${ }^{7}$, and D. Zimmermann ${ }^{9}$

\footnotetext{
${ }^{1}$ Radboud University Nijmegen, Department of Astrophysics, IMAPP, PO Box 9010, 6500 GL Nijmegen, The Netherlands e-mail: petrovic@astro.ru.nl

${ }^{2}$ Institut für Kernphysik, Forschungszentrum Karlsruhe, 76021 Karlsruhe, Germany

3 Institut für Prozessverarb. und Elektr., Forschungszentrum Karlsruhe, 76021 Karlsruhe, Germany

4 ASTRON, 7990 AA Dwingeloo, The Netherlands

5 National Institute of Physics and Nuclear Engineering, 7690 Bucharest, Romania

6 Dipartimento di Fisica Generale dell'Università, 10125 Torino, Italy

7 Max-Planck-Institut für Radioastronomie, 53010 Bonn, Germany

8 Institut für Experimentelle Kernphysik, Universität Karlsruhe, 76021 Karlsruhe, Germany

9 Fachbereich Physik, Universität Siegen, 57068 Siegen, Germany

10 Istituto di Fisica dello Spazio Interplanetario, INAF, 10133 Torino, Italy

11 Fachbereich Physik, Universität Wuppertal, 42097 Wuppertal, Germany

12 Radioastronomisches Institut der Universität Bonn, 53121 Bonn, Germany

13 Soltan Institute for Nuclear Studies, 90950 Lodz, Poland
}

Received 31 May 2006 / Accepted 21 August 2006

\section{ABSTRACT}

\begin{abstract}
Aims. The capability of radio antenna arrays to measure cosmic ray air showers with very large zenith angles is explored. This is important, since a possible neutrino detection has to fulfill two requirements. First: antennas should be able to detect very inclined cosmic ray air showers, and second: it should be possible to estimate the distance to the shower maximum, since neutrinos are most likely to travel far through the Earth's atmosphere without interaction and induce air showers close to the ground.

Methods. LOPES (LOFAR PrototypE Station; LOFAR - LOw Frequency ARray), an array of dipole antennas, is used for the detection of inclined cosmic ray air showers. LOPES is co-located and triggered by the KASCADE (KArlsruhe Shower Core and Array DEtector) experiment, which also provides information on air shower properties such as electron and muon numbers on the ground, as well as the arrival direction. Radio emission of nearly vertical cosmic ray air showers has been detected by LOPES.

Results. LOPES-10 (the first phase of LOPES, consisting of 10 antennas) detected a significant number of cosmic ray air showers with a zenith angle larger than $50^{\circ}$, and many of these have very high radio field strengths. The most inclined event that has been detected with LOPES-10 has a zenith angle of almost $80^{\circ}$. This is proof that the new technique is also applicable for cosmic ray air showers with high inclinations, which in the case that they are initiated close to the ground, can be a signature of neutrino events. Conclusions. Our results indicate that arrays of simple radio antennas can be used for the detection of highly inclined air showers, which might be triggered by neutrinos. In addition, we found that the radio pulse height (normalized with the muon number) for highly inclined events increases with the geomagnetic angle, which confirms the geomagnetic origin of radio emission in cosmic ray air showers.
\end{abstract}

Key words. elementary particles - radiation mechanisms: non-thermal - methods: data analysis - neutrinos

\section{Introduction}

When cosmic rays interact with particles in the Earth's atmosphere, they produce a shower of elementary particles propagating towards the ground almost at the speed of light. The first suggestion that these air showers can produce radio emission was given by Askaryan (1962) based on a charge-excess mechanism. In some experiments during the sixties and seventies, coincidences between radio pulses and cosmic ray events were reported (Jelley 1965; Allan 1971). Recently, Falcke \& Gorham (2003) suggested that the mechanism for a radio emission of air showers might be geosynchrotron emission: secondary electrons and positrons produced in the particle cascade are deflected in the Earth's magnetic field producing radiation 
that is relativistically beamed in the forward direction. They suggested that this radiation can be observed in the radio domain with the new generation of digital radio telescopes like LOFAR (LOw Frequency ARray, Röttgering 2003) and SKA (Square Kilometer Array, Beck 2005). The shower in its densest region has a thickness that is smaller than the wavelength of radio emission below $100 \mathrm{MHz}$ (around a few meters). The emission is then coherent and the signal is amplified. The fact that air showers can be observed in radio frequencies was recently confirmed by the first results of the LOPES (Falcke et al. 2005) and CODALEMA experiments (Ardouin et al. 2005).

An air shower develops in three stages: an initial rapid buildup due to the cascade process, culminating in a particle number maximum, moving with relativistic speed. At the maximum, ionization losses of electrons and positrons roughly equal their $\gamma$-ray production through bremsstrahlung (at a critical energy of about $80 \mathrm{MeV}$ in air). The third phase is a gradual decay as the electrons further lose energy through ionization.

Probably the most important task for radio and particle detectors in cosmic ray physics is finding the composition and primary energy spectrum of cosmic rays, especially at ultra-high energies (UHECR). Currently, it is believed that cosmic rays (protons and nuclei) up to about $10^{15} \mathrm{eV}$ gain their energy in acceleration by shocks from supernova explosions (see, for example, Nagano \& Watson 2000). For cosmic rays with even higher energies it has been suggested that acceleration can happen in the interaction with multiple supernova remnants (Ip \& Axford 1991), galactic wind (Völk \& Zirakashvili 2004), or extragalactic sources like jets of gamma-ray bursts (Waxman 1995; Vietri 1995) or active galactic nuclei (Biermann \& Strittmatter 1987; Rachen \& Biermann 1993). Another option is so-called top-down scenarios: protons and neutrons, but also neutrinos and gamma-rays, are produced from quark and gluon fragmentation of heavy exotic particles formed in the early Universe (Hill 1983; Schramm \& Hill 1983). The highest energy cosmic rays are expected to be suppressed by interaction with the cosmic microwave background radiation, which is known as the Greisen-Zatsepin-Kuzmin (GZK) cut-off (Greisen 1966; Zatsepin \& Kuzmin 1966). The HiRes experiment (Abbasi et al. 2004) measurements are in agreement with the predicted flux suppression of the highest energy cosmic rays. However, the results of the AGASA experiment (Takeda et al. 1998) show an excess compared to the predicted GZK suppression (Bahcall \& Waxman 2003). The ultra-high energy cosmic ray flux is expected to be accompanied by associated fluxes of gamma rays and neutrinos from pion decays formed in the collision of protons with photons (see, for example, Zas 2005).

To reconstruct the initial energy of a primary particle (by measuring the particles on the ground with an array of particle detectors) it is necessary to have a good estimate of the total number of muons and electrons/positrons during shower developments. Muons suffer insignificant losses in the Earth's atmosphere and almost all reach the ground, so they are detected by particle detectors like KASCADE (KArlsruhe Shower Core and Array DEtector, see Antoni et al. 2003). On the other hand, electrons are absorbed proportionally to the shower path length through the atmosphere, and, in case of highly inclined showers, only a small fraction reaches the ground, but the total number of electrons is expected to be roughly proportional to the strength of the radio pulse detected with radio arrays such as LOPES (LOFAR PrototypE Station). The numbers of muons and electrons in the air shower can also reveal the nature of the primary particle. Cosmic ray air showers triggered by nuclei or protons can be recognized by the strong hadronic component, and since muons travel through the atmosphere almost unaffected, a large number of muons can be detected on the ground level. Air showers that are initiated by gamma rays are dominated by the electromagnetic component (electrons, positrons, and photons) while the number of muons is lower.

Neutrinos are not very likely to interact within the Earth's atmosphere and produce air showers due to the very small cross section for interactions. The probability for interaction increases with the path length through the atmosphere, so neutrinos that are most likely to trigger air showers are those entering the atmosphere almost horizontally, possibly producing very inclined cosmic ray air showers that may be detected in the radio domain. Those showers may be triggered anywhere along the traveling path and also close to the ground (Capelle et al. 1998). The cross sections of neutrinos increase with their energy as well, so the highest energy neutrinos are more likely to be detected, but their expected flux is extremely small. It is interesting to mention that Berezinskii \& Smirnov (1975) suggested that muons created in cosmic ray air showers that started high in the atmosphere can trigger showers close to the ground, and these could appear similar to the neutrino-induced ones. However, with radio observations, due to the low attenuation of radio waves, it should be possible to see two showers in this case: the original one in which secondary muons are created and the secondary one, triggered by one of those muons.

Highly inclined showers are expected to be very well detectable in the radio domain (Gousset et al. 2004; Huege \& Falcke 2005a,b) and they can be very interesting for neutrino searches. In this paper we show that the LOPES-10 radio array (the first phase of LOPES, consisting of 10 antennas) can detect highly inclined cosmic ray air showers which can, in principle, be the signature of primary neutrinos. The data is also used to check the dependency of detection efficiency on muon number, zenith, and azimuth angle. Also, highly inclined events are convenient for further investigation of the previously found correlation (Falcke et al. 2005) between radio pulse strength and geomagnetic angle, due to the larger range of geomagnetic angle values. The paper is organized in the following way: in Sect. 2 we briefly introduce the LOPES experiment. In Sect. 3 we present some highly inclined events detection statistics and show an example event with zenith angle of $50^{\circ}$. In Sect. 4 we show some properties of inclined showers. In Sect. 5 we present events with zenith angles larger than $70^{\circ}$. Our conclusions are given in Sect. 6.

\section{The LOPES experiment}

LOPES (LOfar PrototypE Station, see Horneffer et al. 2004) is a phased array of dipole antennas co-located with the KASCADE experiment (Karlsruhe Shower Core and Array Detector, see Antoni et al. 2003), which provides coincidence triggers for LOPES and well-calibrated information about air-shower properties, like electron number $N_{\mathrm{e}}$, total muon number $N_{\mu \text {,tot }}$, truncated muon number $N_{\mu}$ (muon number within 20-400 m of the shower core), azimuth, and zenith angle of the event. Radio emission of cosmic ray air showers $(40-80 \mathrm{MHz})$ has been detected by LOPES-10 in the frequency range from 40 to $80 \mathrm{MHz}$ as reported by Falcke et al. (2005).

The setup of LOPES-10 consists of 10 inverted V antennas distributed over the KASCADE array, which consists of 252 scintillation detector stations on a uniform grid with $13 \mathrm{~m}$ spacing, electronically organized in 16 independent clusters. Each LOPES antenna is centered between four KASCADE huts in the north-western part of the array. The antenna setup has a 

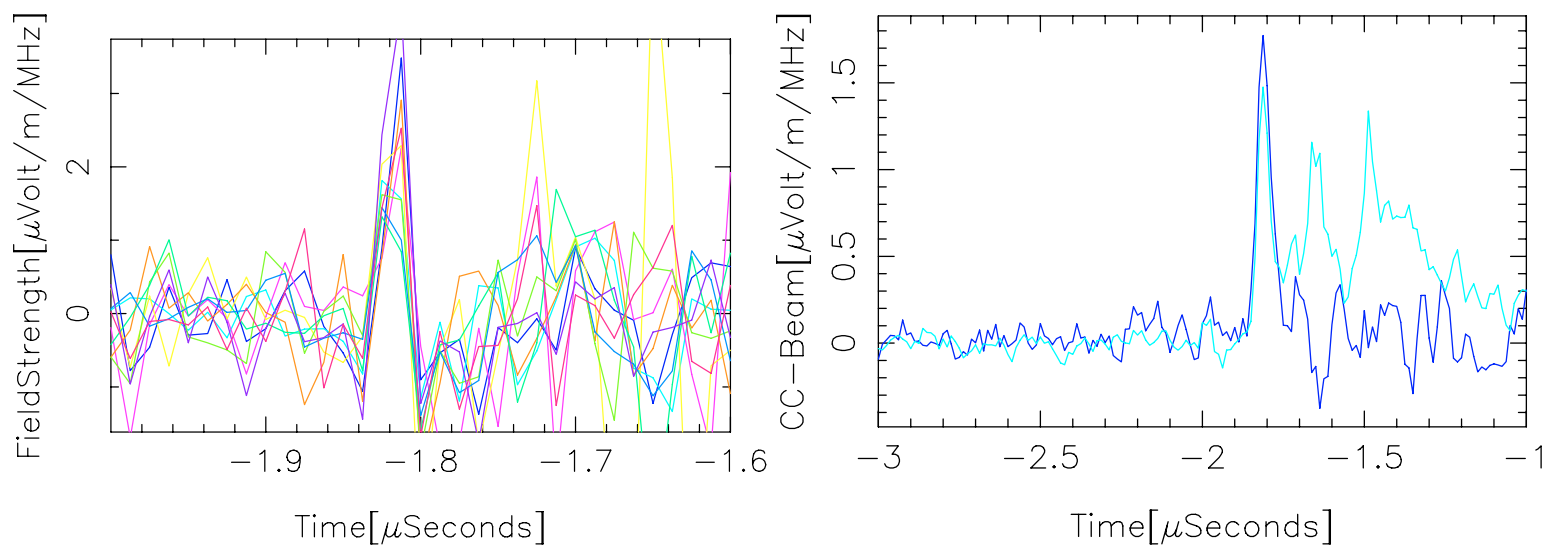

Fig. 1. Left: the electric field as a function of time for LOPES-10 antennas for an event detected in March 2004 with a zenith angle of $53^{\circ}$ and an azimuth angle of $54^{\circ}$. Geomagnetic angle of this event is $70^{\circ}$. Number of electrons is $N_{\mathrm{e}}=1.5 \times 10^{6}$ and total number of muons $N_{\mu \text {,tot }}=1.5 \times 10^{6}$, reconstructed by KASCADE. Right: radio emission as a function of time after beam forming for the same event. The dark blue line represents the resulting cc-beam, the light blue line the power-beam. Note that the timescales on the panels are different.

maximum baseline of $125 \mathrm{~m}$. The analogue radio signal received by antennas is filtered to select a band from 43 to $76 \mathrm{MHz}$, giving a bandwidth of $33 \mathrm{MHz}$.

About $0.8 \mathrm{~ms}$ of data are read out whenever KASCADE produces a "large-event trigger", which means that 10 out of 16 KASCADE clusters have produced an internal trigger. The resulting trigger rate is about two to three per minute (depending on how many clusters are operating and local atmosphere conditions, such as pressure). On average the trigger is delayed with respect to the shower by $1.8 \mu$ s, depending on the shower geometry relative to the KASCADE detector.

We relate the radio signal to shower properties using parameters provided by the KASCADE data processing from the particle detectors. These parameters are: location of the shower core, shower direction, energy deposited in the particle detectors, total number of electrons on the ground level $N_{\mathrm{e}}$, and muon numbers $\left(N_{\mu, \text { tot }}, N_{\mu}\right)$. The strength of the detected radio pulse is in units $\mu \mathrm{V} / \mathrm{m} / \mathrm{MHz}$. There is no absolute gain calibration yet. The positions of radio flashes are coincident with the direction of the shower axis derived from KASCADE data within the expected uncertainties (average offset $0.8 \pm 0.4^{\circ}$ ). Details about the LOPES-10 experiment and data processing are given by Horneffer et al. (2004) and Horneffer (2006).

\section{Radio detection of inclined events}

LOPES-10 received 2017 triggers from the KASCADE detector for events with a zenith angle larger than $50^{\circ}$ in seven months of taking data during the year 2004, with primary energy approximately in the range $10^{16}-10^{17} \mathrm{eV}$ (which is roughly the threshold for the radio detection; the KASCADE detector is optimized for $10^{15} \mathrm{eV}$ ). There were 1931 triggers for events with a zenith angle between $50^{\circ}$ and $70^{\circ}$ and, out of those, roughly 1 in 35 have been detected in the radio domain. With the increase of zenith angle, the number of triggers decreases and there were only 86 events for zenith angles larger than $70^{\circ}$. Out of those events 4 have been detected by LOPES-10. This detection rate is significantly higher than the average detection rate for LOPES10 , which is roughly 1 event detected in the radio domain per 1000 triggers received from the KASCADE detector. The reason for this is that inclined showers that are detected by KASCADE still have a significant number of particles reaching the ground, even after traveling path lengths through the Earth's atmosphere several times longer than the vertical ones. In other words, inclined showers that manage to reach the ground must have high energy primary particles, and they are above the threshold for radio detection. It is also important to mention here that the radio signal of highly inclined events has a larger "footprint" (Huege $\&$ Falcke 2005b) on the ground and due to this the effective collecting area of a radio antenna array becomes bigger. This might be not so important for the small baseline arrays like LOPES-10 (around $100 \mathrm{~m}$ ), but it will be for larger arrays like LOFAR or Pierre Auger Observatory (Nitz \& The AUGER Collaboration 2000).

To reduce low energy air showers triggering KASCADE, for the following detailed investigation of individual inclined cosmic rays, we introduce an additional condition: only showers with large densities in the muon counters are selected. This narrows the sample to 51 events and around $40 \%$ of those are detected by LOPES, many with very large radio pulse heights. The KASCADE detector is not optimized for large zenith angles, so the reconstruction of the electron and muon number is not accurate for all 51 events. However, it is important to mention here that shower direction and shower core position are always well reconstructed by KASCADE.

As an example, we show one of those 51 events (with accurate KASCADE electron and muon number reconstruction), detected by LOPES-10 in March 2004, with a zenith angle of $53^{\circ}$ and an azimuth angle of $54^{\circ}$ (an azimuth of $0^{\circ}$ corresponds to a direction towards the north), with roughly the same number of electrons and total number of muons $N_{\mathrm{e}}, N_{\mu \text {,tot }} \approx 1.5 \times 10^{6}$. The angle between the shower axis and the Earth's magnetic field or so-called geomagnetic angle is $70^{\circ}$. The energy of the primary particle is estimated to be around $10^{17} \mathrm{eV}$.

In Fig. 1 (left panel) we show the electric field (defined as electric field strength divided by the LOPES bandwidth) as a function of time for each of ten LOPES-10 antennas, after being corrected for instrumental and geometric time delays in the direction of the shower axis. The field is coherent at $-1.825 \mu \mathrm{s}$, which is the arrival time of the shower. The incoherent noise afterwards is radio emission from photomultipliers and in this case is relatively weak, indicating a lower energy deposit in the photomultipliers and a lower electron number on the ground than in the case of more vertical events, due to the absorption along the shower's traveling path in the Earth's atmosphere. The right panel of this figure shows the radio emission as a function of time after power-beam and cc(cross correlated)-beam forming. For beam forming, for a given direction, signals are corrected for a time delay between the arrival of signals to each antenna 


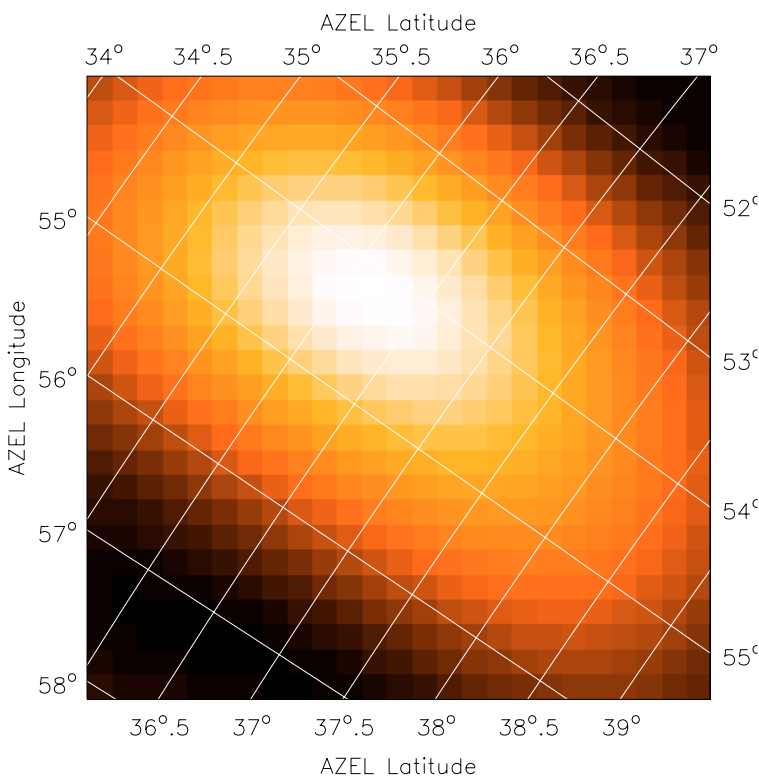

Fig. 2. Radio map of the cosmic ray shower with a zenith angle of $53^{\circ}$ detected in March 2004. Azimuth (AZEL longitude) and elevation (AZEL latitude) of the event are given on the axes. The map is focused on a distance of $3000 \mathrm{~m}$.

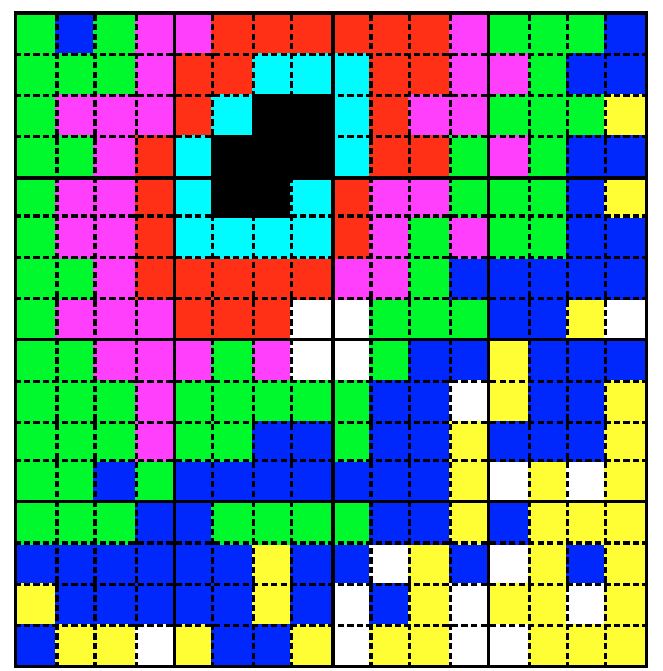

energy deposit [MeV $\left./ \mathrm{m}^{\mathrm{*}}\right]$

Fig. 3. Energy deposit of the cosmic ray shower with a zenith angle of $53^{\circ}$ detected in March 2004 for e/ $\gamma$ KASCADE detectors (area $200 \times 200 \mathrm{~m}^{2}$, each small square represents one KASCADE station). Dark blue color shows the energy deposit of $100 \mathrm{MeV} / \mathrm{m}^{2}$, red color shows $1000 \mathrm{MeV} / \mathrm{m}^{2}$. The maximum energy deposit for this event is $\sim 4500 \mathrm{MeV} / \mathrm{m}^{2}$.

and for antenna gain. Then, the power beam is calculated as follows: the electric field of each antenna is squared, all is summed and normalized by the number of antennas, and the square root is taken. The cross correlated beam is formed in the following way: electric field strengths of all possible two antenna combinations are multiplied, summed, and normalized by the number of antennas, and finally, the square root is taken (while preserving the sign). The power beam gives a peak if there is a lot of power in the antennas, independent of it being coherent or incoherent, while the cc-beam is more sensitive to the coherence effects (for more details see Horneffer 2006).

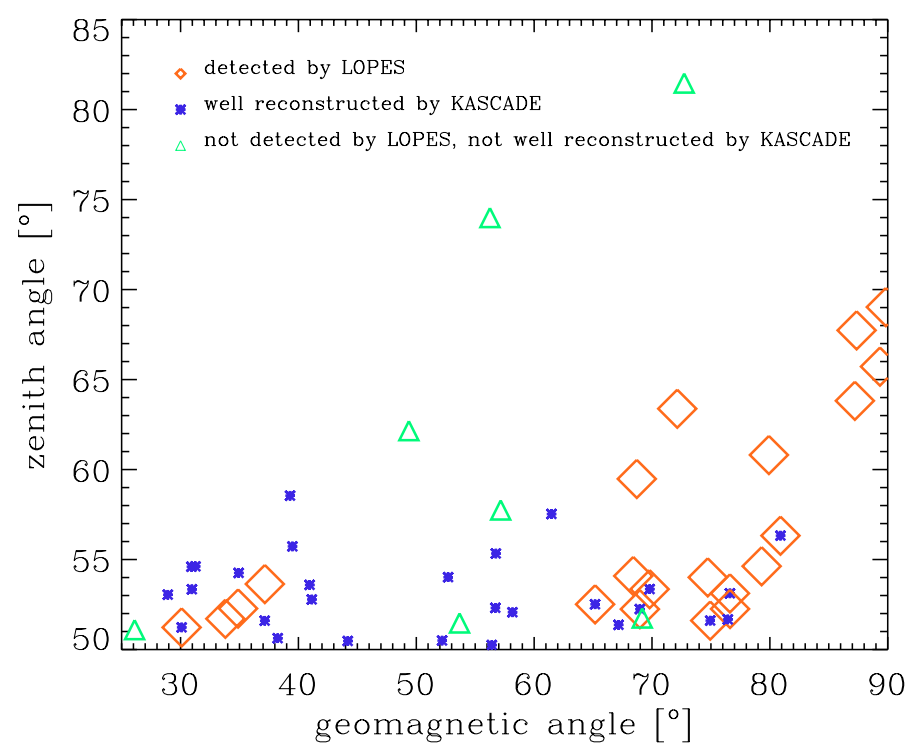

Fig. 4. Geomagnetic and zenith angle of inclined events from the year 2004. Rhombs: events detected by LOPES-10 in the radio domain, stars: events with the reliable KASCADE reconstruction of muon and electron number, and triangles: events not detected by LOPES and without well reconstructed muon and electron numbers by KASCADE. Radio detection efficiency is almost $100 \%$ above a geomagnetic angle of $60^{\circ}$.

Figure 2 is a radio map of the example event (Falcke et al. 2005). The air shower is the brightest point in the sky at the radio frequencies for several tens of nanoseconds. Figure 3 gives the energy deposit of the chosen cosmic ray shower over the KASCADE array with e/ $\gamma$ detectors. We can see that the shower core falls within the KASCADE array and that the maximum energy is deposited in the north-western part, within the LOPES10 array. We can notice the elliptical shapes of the contours of the energy deposit, which is typical for inclined events.

We should also point out that the antenna gain (ratio of the power received/transmitted in a certain direction to the power that an isotropic emitter would radiate in that direction) decreases with an increase of zenith angle (the gain also depends on frequency and azimuth), so the sensitivity of LOPES-10 decreases towards the horizon. For example at fixed azimuth angle, the gain is around three times larger for a zenith angle of $20^{\circ}$ than for a zenith angle of $70^{\circ}$. We should keep in mind that due to this, on a map of an inclined event, (for example Fig. 2), the maximum can be slightly shifted towards lower zenith angles. Also, gain values for larger zenith angles are less certain.

\section{Properties of inclined showers}

Out of 51 events with a zenith angle larger than $50^{\circ}$, two occurred in thunderstorm conditions, and are taken out of the further analysis (Buitink et al. 2005). The remaining 49 events are shown in Figs. 4 and 5.

Figure 4 shows geomagnetic and zenith angles of inclined events triggered by the KASCADE detector in the year 2004. Out of those, 28 events have accurately reconstructed muon and electron numbers by KASCADE (stars), and 21 were detected by LOPES-10 (rhombs). Note that there are 7 events with accurate KASCADE reconstruction that are detected by LOPES-10. Other events (21) reconstructed by KASCADE are not registered in the radio domain. Also, there are 14 events "seen" by LOPES-10, but with no reliable particle numbers from KASCADE. Finally, there are 7 events not accurately 


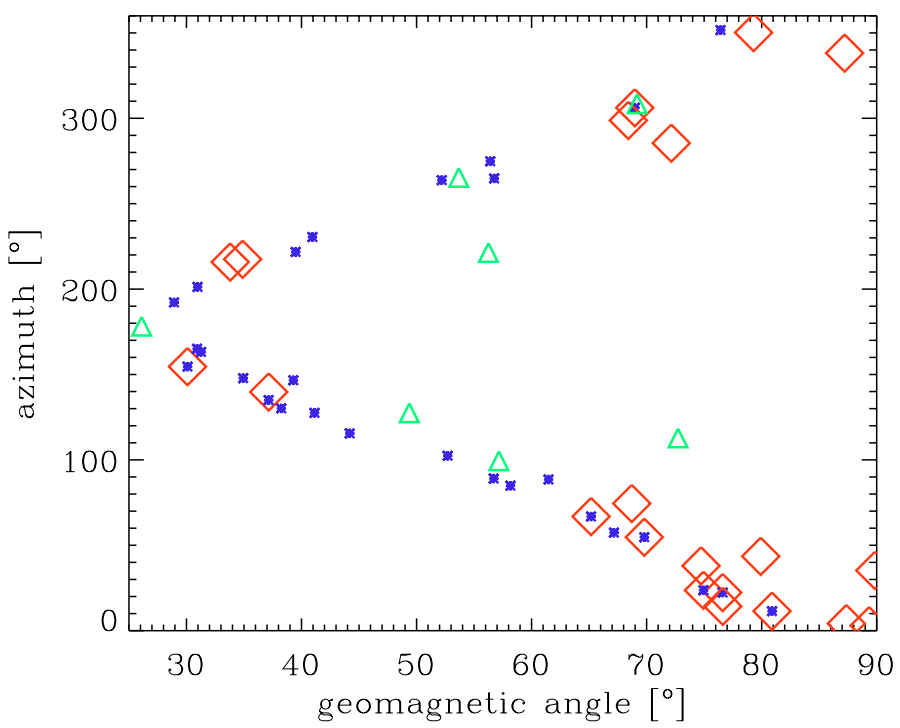

Fig. 5. Geomagnetic angle and azimuth for the same set of events as in Fig. 4. The meaning of symbols is the same as in Fig. 4. Note that there are more events detected by LOPES-10 in the north-south direction than in the east-west direction. This may be due to the antenna sensitivity, largest geomagnetic angles in the direction of north or polarization effects.

reconstructed by KASCADE and also not detected by LOPES10 (triangles).

We can see that above a zenith angle of $60^{\circ}$, muon and electron number sreconstructed by KASCADE are not reliable, due to the detector sensitivity decrease for large inclinations. On the other hand, the most inclined cosmic ray air shower (registered as highly energetical with a large muon density on the ground) detected in the radio domain has a zenith angle of almost $70^{\circ}$. Note that almost all events with a large geomagnetic angle (more than $60^{\circ}$ ) are detected by LOPES-10. This corresponds to a direction towards the north. Events detected by LOPES with geomagnetic angles between $30^{\circ}$ and $40^{\circ}$ are in the southern direction.

The same can be seen in Fig. 5. It shows the geomagnetic angle and azimuth of 49 inclined events. Symbols have the same meaning as in the Fig. 4. An azimuth of $0^{\circ}$ corresponds to a direction towards the north. All radio detected events are $\pm 80^{\circ}$ around the north and $\pm 40^{\circ}$ around the south. Events from east and west directions are not detected. This may be due to two times larger antenna sensitivity in the north-south than in the east-west direction for inclinations larger than $50^{\circ}$ and/or due to the fact that showers coming from east or west are expected not to have a significant emission in the east-west polarization component (Huege \& Falcke 2005b), which is the one that LOPES antennas measure.

There are more detected events coming from the north than from the south, which is probably related to the fact that cosmic rays coming from the north have the largest geomagnetic angles. Also, the tight correlation (two thin bands) shown in Fig. 5 is due to the fact that most events have zenith angles between $50^{\circ}$ and $60^{\circ}$, so the azimuth changes from $0^{\circ}$ to $360^{\circ}$, whereas the zenith angle stays almost constant for those events. The only two points out of this general trend are for zenith angles larger than $70^{\circ}$.

Figure 6 shows events with zenith angles smaller than $50^{\circ}$ (Horneffer 2006) and inclined events from this paper with accurate KASCADE particle number reconstruction, which are in the region of $100 \%$ radio detection considering their geomagnetic angles and truncated muon numbers. This can be explained as

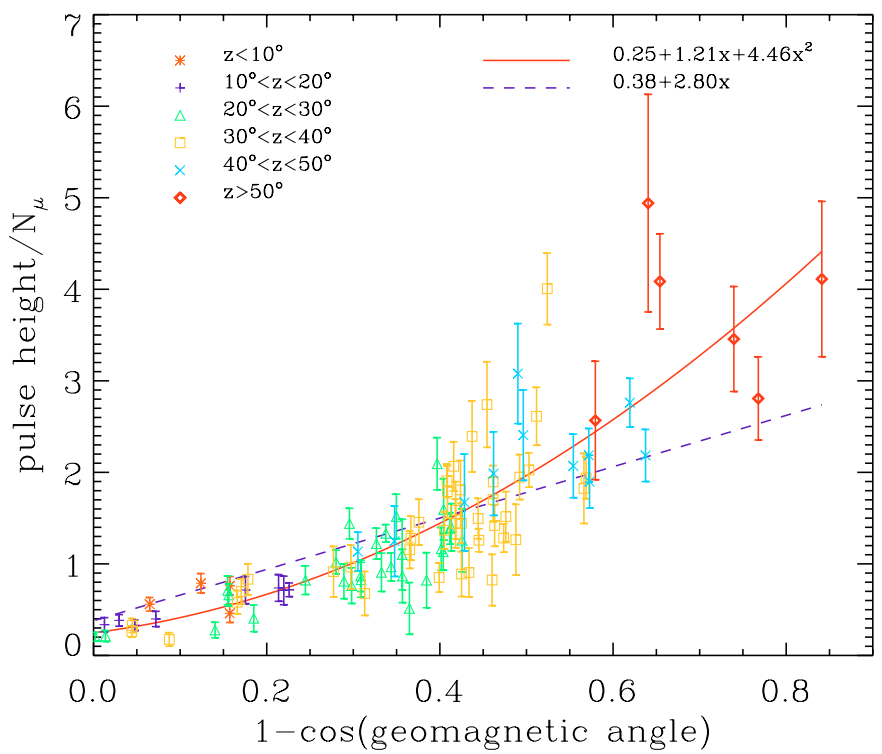

Fig. 6. Radio pulse normalized with the truncated muon number (and multiplied with $10^{6}$ ) as a function of a geomagnetic angle for bright events (Horneffer 2006) and inclined events from this paper in the regime of $100 \%$ radio detection. The dashed line represents the linear fit for bright events with $z<50^{\circ}$, and the solid line represents the quadratical fit for all events.

follows: the fraction of radio detected events within KASCADE triggered events increases both with rising muon number and with rising geomagnetic angle. By defining a more strict cut on muon number and geomagnetic angle, 1-cos(geomagnetic angle) $>3.58-0.6 \log \left(N_{\mu}\right)$, as explained in detail by Horneffer (2006), it is possible to get a selection where all events have been detected in the radio domain. Figure 6 shows the correlation between the radio pulse normalized by the truncated muon number and the geomagnetic angle. The normalized radio pulse height increases with the geomagnetic angle, for highly inclined events also, which indicates the geomagnetic origin of shower radio emission. The value used for the radio pulse height in Fig. 6 is the height of a Gaussian fitted to cc-beam and this value is affected by errors of the KASCADE data and statistic and systematic errors connected with radio measurements (Horneffer 2006). We should point out that the gain of the LOPES antennas is calculated from the simulations and that the real gain can differ from those values. The difference is the largest for large zenith angles, due to the unknown reflections at the ground and the surroundings. We conclude from the simulations that for zenith angles less than $50^{\circ}$, the effect on the pulse height is less than $10 \%$ (Horneffer 2006). The corresponding estimated error for zenith angles larger than $50^{\circ}$ is up to $50 \%$. In Fig. 6, beside other errors, we included $10 \%$ gain error for events with $z<50^{\circ}$ and $50 \%$ gain error for more inclined events. In this way, we took the largest possible value of the error for highly inclined events into the account. We made a linear fit for events $z<50^{\circ}$ and a quadratical fit for all events. We can notice that even with a maximum gain error included, events with $z>50^{\circ}$ are located above the linear fit and match the quadratical dependence more closely. However, we should keep in mind that a larger number of events with a large zenith angle might be necessary for a final conclusions about this dependence, and also, that LOPES-10 antennas measure only east-west polarization and this may be the reason that events coming from the north have larger measured radio pulse heights. 


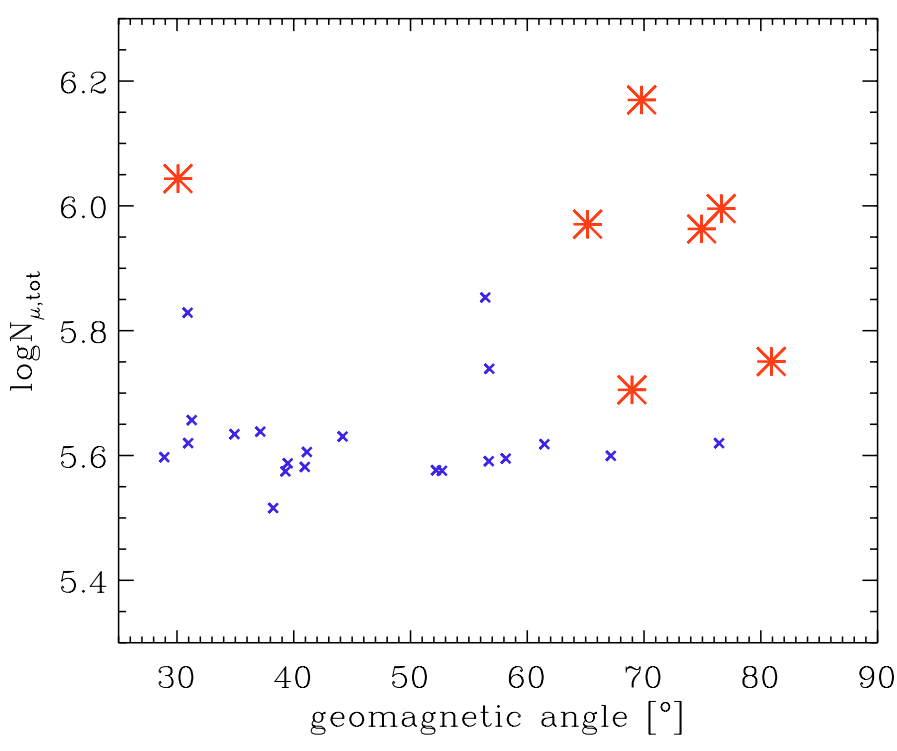

Fig.7. Geomagnetic angle and the total number of muons (on the ground level) for inclined events from 2004 that have reliable KASCADE reconstruction of muon and electron number. Big stars represent events detected by LOPES-10. Events with large geomagnetic angles and/or large muon numbers are favored for detection in the radio domain.

Figure 7 shows the geomagnetic angle and the total muon number (on ground level) of 28 inclined events with accurate KASCADE electron and muon numbers. Out of those, 7 events were detected by LOPES-10 (large stars). Radio detection clearly favors cosmic ray air showers with a large muon number, which is in agreement with bright, nearly vertical events from Falcke et al. (2005). This is expected, since theory predicts that due to coherence (Huege \& Falcke 2003), the electric field strength scales approximately linearly with the number of electrons/positrons (times their track length) in the shower, which is roughly proportional to the number of muons in the case of the KASCADE detector.

\section{Events with a zenith angle larger than $70^{\circ}$}

If we search through $86 \mathrm{KASCADE}$ triggers for events with a zenith angle larger than $70^{\circ}$, we find four events that are detected in the radio domain by LOPES-10. We do this without selecting events with a large muon density on the ground, and that is why they do not show on plots in the previous section. This does not mean that the number of particles in those showers is small, but due to the very large inclination, muon and electron numbers are difficult to reconstruct with the KASCADE detector.

We show two examples here. The left panel of Fig. 8 shows an event from January 2004. We checked and ruled out the possibility that this event occurred in a thunderstorm enviroment (Buitink et al. 2005). This is the most inclined radio event detected by LOPES-10 during the year 2004, with a zenith angle of $77^{\circ}$ and an azimuth of $317^{\circ}$. The geomagnetic angle for this event is $84^{\circ}$, which is again in agreement with the conclusion we have drawn earlier that events with a large geomagnetic angle are favored for radio detection. The signal is coherent with a very large field strength. Note that the noise after the peak is very weak, due to the low noise from the photomultipliers, i.e., a small number of electrons that reach the ground, which is expected in the case of very inclined events. A power-beam and a cc-beam formed pulses are shown in the right panel of Fig. 8.
Another event with a zenith angle larger than $70^{\circ}$ is shown in Fig. 9. Here, the thunderstorm connection is also ruled out. This event is from July 2004 with a zenith angle $73^{\circ}$, an azimuth of $300^{\circ}$, and a geomagnetic angle of $88^{\circ}$.

\section{Conclusions}

We have made a selection of events detected by LOPES-10 during the year 2004 with a zenith angle larger than $50^{\circ}$ and a large muon density on the ground, which corresponds to a high energy of the primary particle (51 events). Around $40 \%$ of those events are detected in the radio domain, and some of them with very high field strengths, like the example we presented in Sect. 3. We also found four radio detected events with a zenith angle larger than $70^{\circ}$ (Sect. 4).

We found that inclined events that are preferred for radio detection are the ones with a large geomagnetic angle and/or a large number of muons, which is proportional to the total number of particles in a shower, i.e., the energy of the primary particle. We also found that the radio pulse height normalized with the truncated muon number increases with the geomagnetic angle to larger zenith angles than considered in Falcke et al. (2005). This implies that geomagnetic processes in cosmic ray air showers are also dominant emission mechanism for highly inclined events.

For the particle detector KASCADE, the sensitivity drops sharply towards the larger zenith angles. However, this is not the case for radio detection. Our results show that radio arrays can be used to detect very inclined cosmic ray air showers. We show that LOPES-10 can detect events up to a zenith angle of around $80^{\circ}$. This indicates that much larger radio detectors, if they are able to estimate the distance to the air shower maximum, might be used in the future as a possible detectors of neutrino induced showers with large inclinations initiated close to the ground.

However, for a possible future detection of neutrinos with a large radio array, like the LOFAR telescope, we should keep in mind the following things:

- Detection efficiency of the LOPES-10 radio array depends strongly on the geomagnetic angle and also, due to antenna sensitivity and polarization effects, on the azimuth angle. This makes certain directions favorable for radio detection and this is an important issue considering the very low flux of high energy neutrinos. For further understanding of geomagnetic angle dependence of a radio signal, it is necessary to understand better the emission processes in cosmic ray air showers. Azimuth angle detection efficiency dependence can possibly be solved by using antennas that measure both polarization directions.

- LOPES-10 data do not show radio detected events above $80^{\circ}$, possibly because of the missing triggers from the KASCADE array. In addition, the LOPES-10 antenna gain decreases towards the horizon and is not accurately known for large zenith angles. Knowing the exact effect of the radio wave reflections on the ground and surroundings (now estimated to be up to $50 \%$ on radio pulse height measured by LOPES-10 for highly inclined events) would greatly improve the estimate of the antenna gain at large zenith angles. Another possible way to reduce this problem is to use an optimized antenna for measuring inclined showers, but it is important to remember that one has to make a trade-off between antenna sensitivity to the horizon and suppression of human-made radio emission, which usually comes from the horizon and decreases the signal-to-noise ratio even after beam forming. 

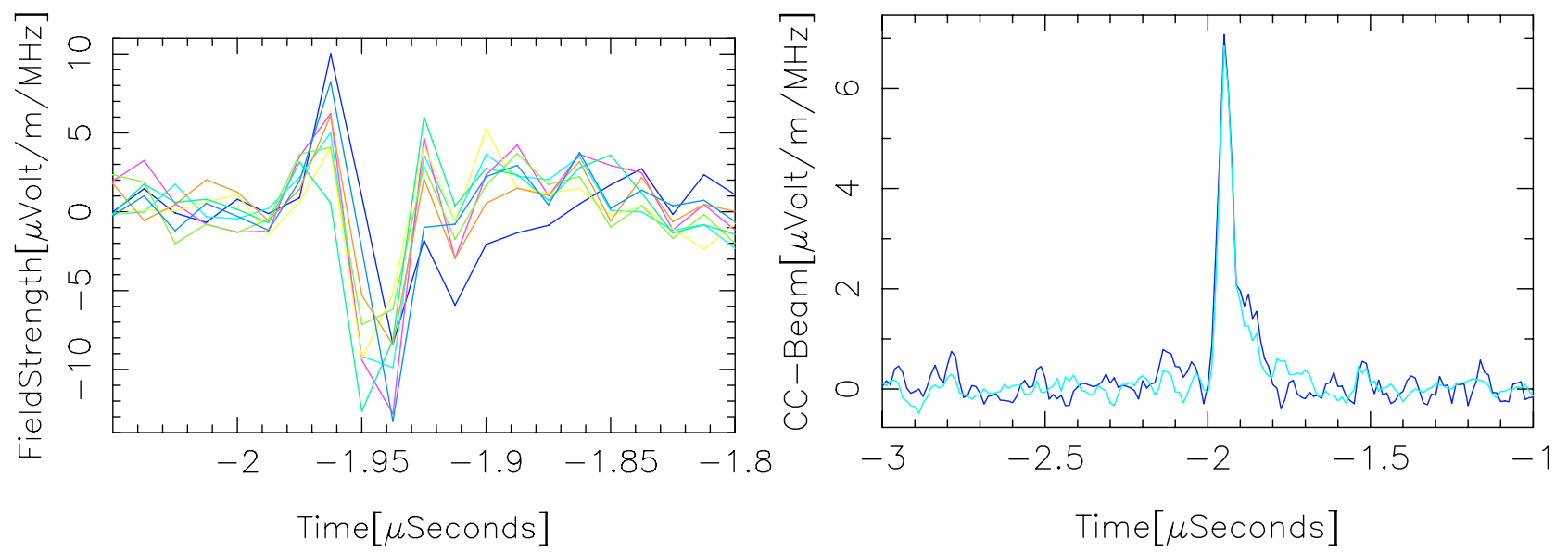

Fig. 8. Left: Electric field as a function of time for LOPES-10 antennas for an event detected in January 2004 with a zenith angle of $77^{\circ}$. The geomagnetic angle is $84^{\circ}$. Right: Radio emission as a function of time after beam forming for the same event. The dark blue line represents cc-beam, the light blue line the power-beam. Note that the timescales on the panels are different.
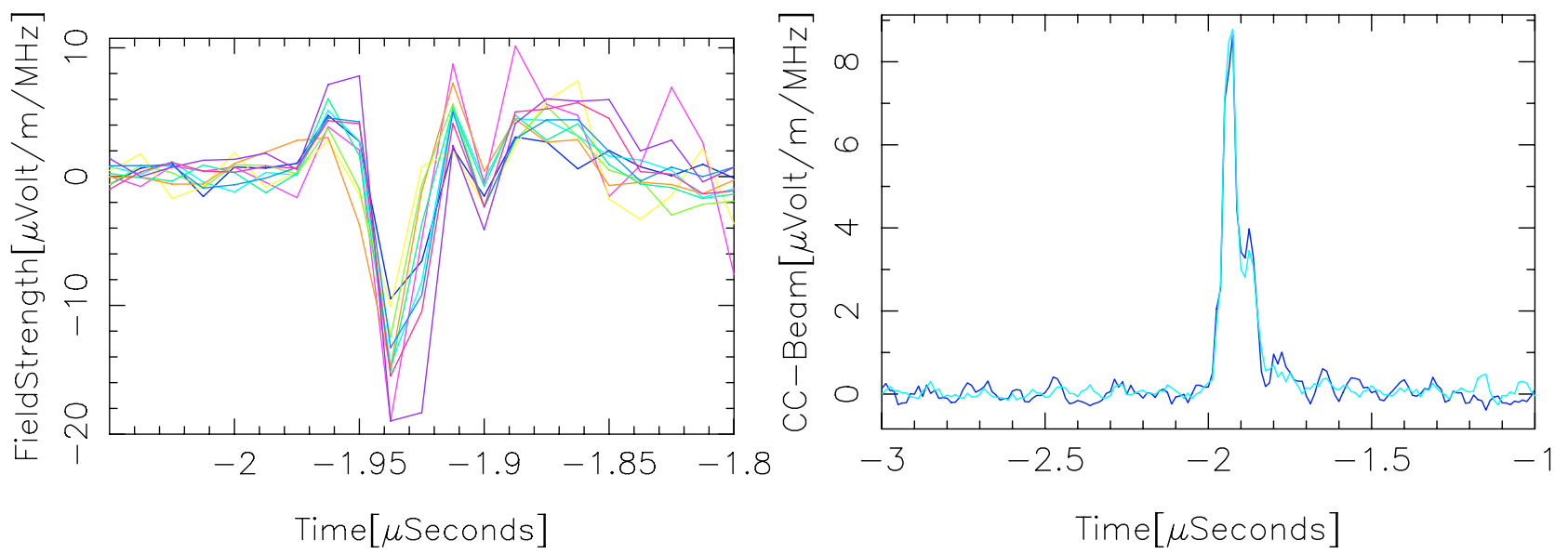

Fig. 9. Left: electric field as a function of time for LOPES-10 antennas for an event detected in July 2004 with a zenith angle of $73^{\circ}$. The geomagnetic angle is $88^{\circ}$. Right: radio emission as a function of time after beam forming for the same event. The dark blue line represents the resulting cc-beam, the light blue line the power-beam. Note that the timescales on the panels are different.

\begin{abstract}
Acknowledgements. This work is part of the research program of the Stichting voor Fundamenteel Onderzoek der Materie (FOM), which is financially supported by the Nederlandse Organisatie voor Wetenschappelijk Onderzoek (NWO). LOPES is supported by the German Federal Ministry of Education and Research. The KASCADE-Grande experiment is supported by the German Federal Ministry of Education and Research, the MIUR of Italy, the Polish Ministry of Science and Higher Education, and the Romanian Ministry of Education and Research (grant CEEX 05-D11-79/2005).
\end{abstract}

\section{References}

Abbasi, R. U., Abu-Zayyad, T., Amann, J. F., et al. 2004, Phys. Rev. Lett., 92, 151101

Allan, H. 1971, Prog. in Element. Part. and Cos. Ray Phys., 10, 171

Antoni, T., Apel, W. D., Badea, F., et al. 2003, Nucl. Instr. Methods Phys. Res. A, 513, 490

Ardouin, D., Belletoile, A., Charrier, D., et al. 2005,

[arXiv: astro-ph/0504240]

Askaryan, G. 1962, Soviet Phys. JETP, 14, 441

Bahcall, J. N., \& Waxman, E. 2003, Phys. Lett. B, 556, 1

Beck, R. 2005, Astron. Nachr., 326, 608

Berezinskii, V. S., \& Smirnov, A. I. 1975, Ap\&SS, 32, 461

Biermann, P. L., \& Strittmatter, P. A. 1987, ApJ, 322, 643

Buitink, S., Apel, W. D., Badea, A. F., et al. 2005, ICRC Proc., Pune, India

Capelle, K. S., Cronin, J. W., Parente, G., \& Zas, E. 1998, Astropart. Phys., 8, 321
Falcke, H., \& Gorham, P. 2003, Astropart. Phys., 19, 477

Falcke, H., Apel, W. D., Badea, A. F., et al. 2005, Nature, 435, 313

Gousset, T., Ravel, O., \& Roy, C. 2004, Astropart. Phys., 22, 103

Greisen, K. 1966, Phys. Rev. Lett., 16, 748

Hill, C. T. 1983, Nucl. Phys. B, 224, 469

Horneffer, A. 2006, Ph.D. Thesis, University Bonn,

[http://nbn-resolving.de/urn:nbn:de:hbz:5N-07819]

Horneffer, A., Antoni, T., Apel, W. D., et al. 2004, in Proc. SPIE, 5500, 129

Huege, T., \& Falcke, H. 2003, A\&A, 412, 19

Huege, T., \& Falcke, H. 2005a, A\&A, 430, 779

Huege, T., \& Falcke, H. 2005b, Astropart. Phys., 24, 116

Ip, W.-H., \& Axford, W. I. 1991, in Astrophysical Aspects of the Most Energetic Cosmic Rays, 273

Jelley, J. 1965, Nature, 205, 327

Nagano, M., \& Watson, A. A. 2000, Rev. Mod. Phys., 72, 689

Nitz, D., \& The AUGER Collaboration. 2000, Nucl. Phys. B Proc. Suppl., 80, C811

Rachen, J. P., \& Biermann, P. L. 1993, A\&A, 272, 161

Röttgering, H. 2003, New Astron. Rev., 47, 405

Schramm, D. N., \& Hill, C. T. 1983, in International Cosmic Ray Conference, 393

Takeda, M., Hayashida, N., Honda, K., et al. 1998, Phys. Rev. Lett., 81, 1163

Vietri, M. 1995, ApJ, 453, 883

Völk, H. J., \& Zirakashvili, V. N. 2004, A\&A, 417, 807

Waxman, E. 1995, Phys. Rev. Lett., 75, 386

Zas, E. 2005, New Journal of Physics, 7, 130

Zatsepin, Z., \& Kuzmin, V. 1966, Zh. Eksp. Teor. Fiz. Pisma Red., 4, 144 